\title{
Enhanced microwave transmission through a patterned metal film
}

\author{
R. J. Kelly, ${ }^{\text {a) }}$ M. J. Lockyear, J. R. Suckling, and J. R. Sambles \\ Electromagnetic Materials Group, School of Physics, University of Exeter, Devon EX4 4QL, \\ United Kingdom \\ C. R. Lawrence \\ Qinetiq, Cody Technology Park, Farnborough, Hampshire GU14 OLX, United Kingdom
}

(Received 19 January 2007; accepted 7 May 2007; published online 31 May 2007)

\begin{abstract}
Selective transmission of radiation through a two-dimensional array of subwavelength slits in an otherwise opaque thin metal film is presented at microwave frequencies. Individual slits are modified with the addition of perpendicular cuts, which interestingly and perhaps counterintuitively leads to resonant transmission when the incident radiation is polarized parallel to the slits. Finite element modeling of the structure shows the transmission of radiation polarized parallel to the slit direction to be a result of induced surface currents exciting a zeroth-order Fabry-Pérot mode.

(C) 2007 American Institute of Physics. [DOI: 10.1063/1.2745202]
\end{abstract}

Electromagnetic (EM) pollution is currently one of the fastest growing forms of pollution due in part to the popularity of the mobile phone as a convenient communication device. As many environments such as hospitals and airports rely increasingly on sensitive electronic equipment, EM screening materials have generated much commercial interest. Indeed, a substantial body of work regarding frequency selective screens (FSSs) based on planar slit geometry exists. $^{1-8}$ However, for this geometry a Fabry-Pérot-like mode is utilized to mediate transmission at well defined frequencies, which imposes a characteristic thickness upon the transmitting structure $\left(\geqslant \lambda_{0} / 2\right){ }^{3}$ Here we present a structure that acts as a lightweight FSS formed from an otherwise opaque ultrathin aluminum film where the metal thickness $t$ is much less than the skin depth $\delta$ at these frequencies.

A one-dimensional array of subwavelength slits in metal will readily transmit radiation via coupled surface waves due to diffraction when the electric vector is polarized perpendicularly to the slit direction. ${ }^{1}$ By contrast, radiation polarized with its electric vector parallel to the slits is reflected. Extending the array into two dimensions introduces fourfold symmetry, allowing transmission that is polarization independent since incident radiation will always have a component of the electric vector orthogonal to one of the slit directions. ${ }^{5}$ If the slit array is deep with respect to the incident wavelength, i.e., $d \geqslant \lambda_{0} / 2$, then it is also possible to couple to a series of Fabry-Perot-type modes, ${ }^{1}$ with the fundamental Fabry-Pérot mode existing at a wavelength corresponding to twice the slit depth. However, Suckling et al. ${ }^{9}$ demonstrated that for incident radiation polarized parallel to the slit direction, it is possible to couple to a "zeroth-order" Fabry-Pérot mode. Here we propose a structure based on a planar subwavelength slit geometry that selectively transmits radiation at millimeter wavelengths via a coupled surface wave and remarkably allows coupling to a zeroth-order Fabry-Pérot mode while having a thickness of $1 / 100$ of the operating wavelength. Coupling to both modes occurs for any azimuthal angle and is polarization independent.

The structures presented here are formed from an array of slits cut into a $40 \mathrm{~nm}$ aluminum film. Despite being less

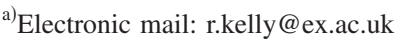

than the skin depth at the operating wavelength, the aluminum remains opaque due to the high impedance mismatch across the metal-air boundary. The film is also backed by a dielectric layer of thickness $100 \mu \mathrm{m}$. The three structures A, $\mathrm{B}$, and $\mathrm{C}$ are shown in Fig. 1. For structure A each unit cell consists of a square patch of metal and is perforated by four "slits" close to each edge. Structures B and C have an additional "cut" directed centrally from each slit and of differing lengths between the two structures. The unit cell has dimensions of $5.9 \times 5.9 \mathrm{~mm}^{2}$, with the slits measuring $0.18 \mathrm{~mm}$. These dimensions are identical for A, B, and C. For structure $B$ the cut width is $0.57 \mathrm{~mm}$ and extends $0.43 \mathrm{~mm}$ toward the center of the patch, tapering to a point over a further $0.40 \mathrm{~mm}$ (the taper allows cuts to extend deep into the sample area). Structure $C$ has an identical cut width and extends $0.99 \mathrm{~mm}$ toward the center of the patch, once again tapering to a point over a further $0.40 \mathrm{~mm}$. Transmission spectra are obtained over the range $18 \mathrm{GHz}<f_{0}<60 \mathrm{GHz}$ by placing samples between matched microwave source and detector horns, with each horn $500 \mathrm{~mm}$ away from the sample. In addition, the incident radiation is reduced to a well-collimated central part by an aperture formed from microwave absorbing material. Data are normalized to spectra obtained from the same setup with the sample removed.

Figure 2 shows transmission as a function of frequency for structures A, B, and C when illuminated by $p$-polarized plane wave radiation at normal incidence. Also shown (solid line) are the model predictions of a finite element method (FEM) model, ${ }^{10}$ which show good agreement even though the model treats the sample as infinite in the surface plane

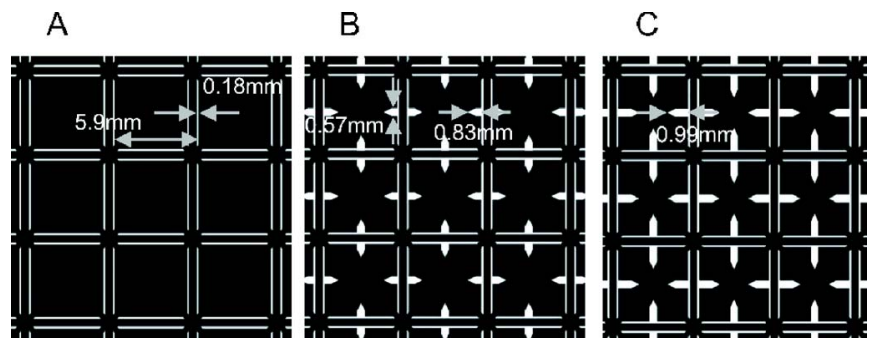

FIG. 1. Structures under investigation. Light areas are where metal has been removed. 

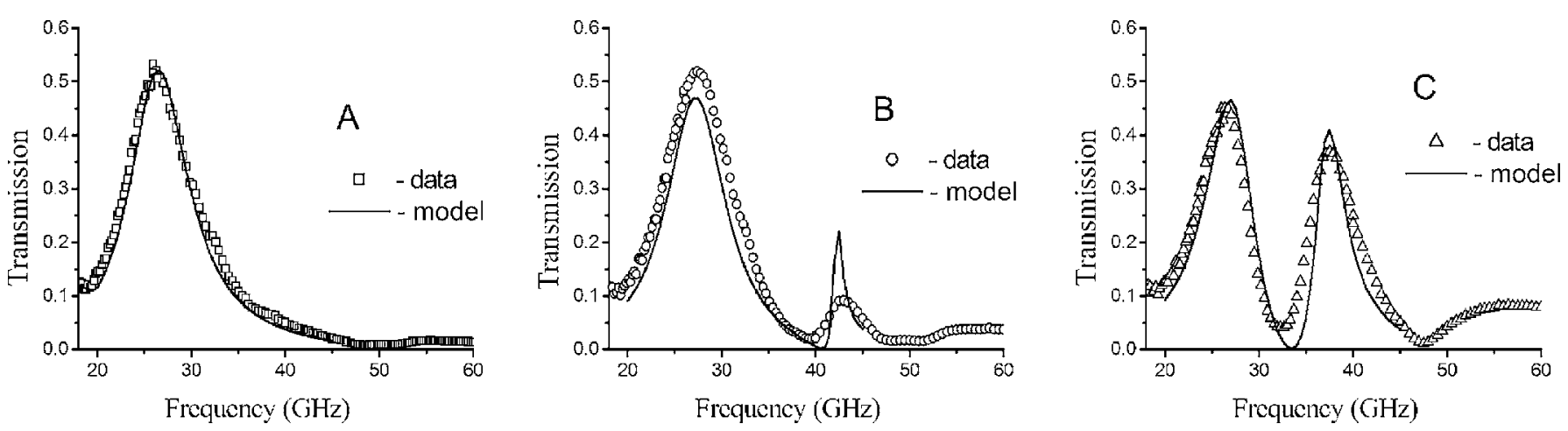

FIG. 2. Transmission data for the three structures. 1 data point in 10 is plotted for clarity. Also plotted is the prediction provided from the FEM code.

and does not take into account experimental beam spread $\left(\sim 1^{\circ}\right)$. Structure A gives a single peak in transmission over the given frequency range which will henceforth be termed "mode 1." Structures B and C exhibit an additional higher frequency transmission peak, termed "mode 2." Since the only difference between the two sets of structures are the cuts, one may attribute this secondary transmission peak to the interaction of the incident field with the cuts. This suggestion is further supported by noting that a change in the cut depth results in a shift in the frequency of the resonant transmission of mode 2 . It may be noted that the prediction from the FEM for mode 2 in structure B differs somewhat from the data in both magnitude and width. This is due to a higher degree of sample variability in the cuts in structure B, causing a flattening and broadening of the resonant mode.

In order to understand the electromagnetic response of these structures it is useful to explore the electromagnetic fields of the structures at resonance using the FEM model. Figure 3 illustrates (a) the time-averaged $E$-field magnitude plotted in the $x y$ plane situated $0.025 \mathrm{~mm}$ from the back face of the structure and (b) the instantaneous $E$ vector plotted in the $y z$ plane through the center of the unit cell in the $x$ direction at a temporal phase corresponding to maximum field enhancement for mode 1, which peaks at $27 \mathrm{GHz}$, and is common to all structures. Fields for mode 1, here plotted for structure A, are only marginally perturbed by the presence of the cuts for structures B and C. Incident radiation is polarized such that the electric vector is perpendicular to the $x$ axis, and regions of high field are denoted by lighter shading. The high fields located beneath the slits perpendicular to the

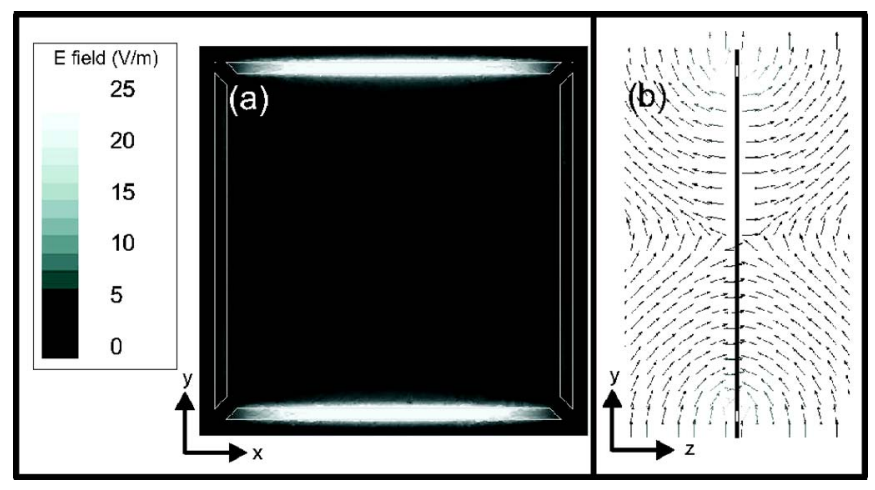

FIG. 3. (a) Time-averaged electric field magnitude for structure A. Incident radiation is polarized with its electric vector perpendicular to the $x$ axis. (b) Electric vector for the same structure showing surface waves on the front and back of the metallic layer. Electric vector is plotted at a phase corresponding to maximum field enhancement. acts primarily as a capacitive region, although the magnitude
Downloaded 23 May 2008 to 10.1.150.176. Redistribution subject to AlP license or copyright; see http://apl.aip.org/apl/copyright.jsp incident polarization direction are consistent with a coupled surface wave on either surface of the metallic layer, and show a 25-fold enhancement factor relative to the injected field in the FEM model. Incident radiation diffractively couples to a surface wave that has a wavelength of twice the grating pitch. Figure 3(b) displays the surface wave, showing the electric field to be quantized along the distance between slits, with a node at the center, having a maximum field located around each slit. A similar field profile is displayed on the back face of the structure. Electric fields are unable to penetrate the metal due to its high conductivity at these frequencies, and therefore couples through the gaps, increasing the field intensity in the vicinity of the slit and exciting a surface wave on the back face of the metal. The surface wave on the back face is then diffracted to an outgoing propagating plane wave of the same frequency, leading to an increased transmittance of the structure at a wavelength equal to that of the surface wave. The presence of the dielectric layer on the back face causes the coupling efficiency of the front and back surfaces to be reduced, as it introduces a slight mismatch in the energies of the respective surface waves. However, the effect is minimal as the dielectric layer is very thin compared to the extent of the fields away from the structure, which is several millimeters at these frequencies.

Field plots for mode 2 in structure B are quite different. Figure 4(a) shows the time-averaged $E$-field magnitude for this structure, again plotted in the $x y$ plane at a distance $0.025 \mathrm{~mm}$ from the back face of the metallic layer, with incident radiation polarized such that the electric vector is perpendicular to the $x$ axis. For this mode high fields (again indicated by lighter shading) are associated with those slits which are parallel to the polarization direction, and the enhancement factor is 15 times that of the injected field in the FEM model. To appreciate how these enhanced fields occur, we must look at other elements of the field interaction with the structure, and how they differ from mode 1. Figure 4(b) shows the surface current density on the front face of the aluminum. Radiation incident on the sample generates surface currents in a direction parallel to the incident electric vector. The cut in each slit perturbs the surface currents, producing partial current loops. As a result, magnetic fields with a 20-fold enhancement are induced within the cuts [Fig. 4(c)] which loop through the adjacent cut in each array element. Thus, the cuts behave primarily as inductive elements. Loops in the surface current around each cut also perturb the surface charge density in the vicinity of the cut. This results in induced electric fields across the slit, and as such the slit 


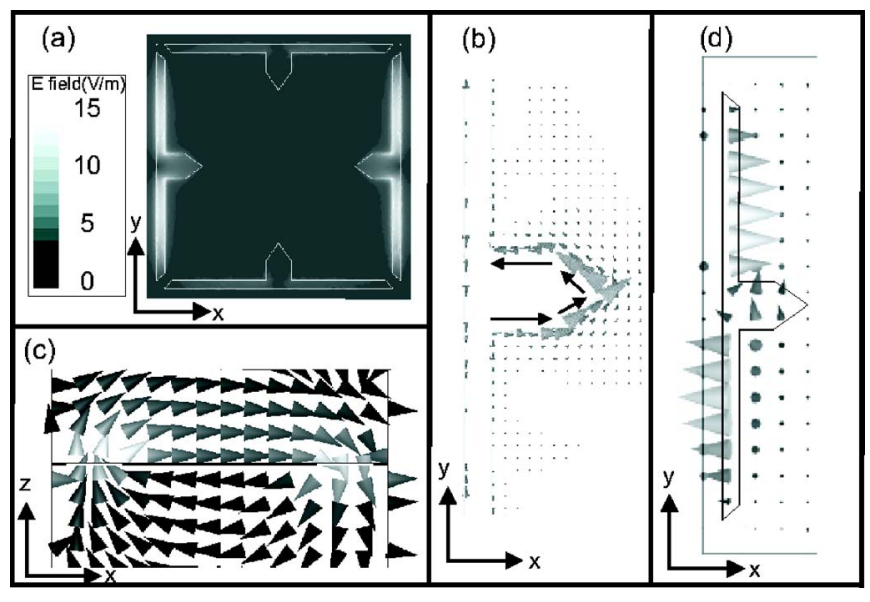

FIG. 4. (a) Time-averaged electric field magnitude for structure B. Incident radiation is polarized with its electric vector perpendicular to the $x$ axis. (b) Surface currents (indicated by arrowheads) are perturbed by the cut and form a partial current loop (arrows with tails are added for clarity). (c) Partial current loops [shown in (b)] induce magnetic field loops (indicated by arrowheads) between adjacent cuts. (d) The electric vector (indicated by arrowheads) within the slit is rotated by $90^{\circ}$ from that of the incident polarization.

of this induced electric field is half that of the incident electric field.

Figure 4(d) shows the instantaneous $E$ vector at a phase corresponding to maximum enhancement plotted in the $x y$ plane at a distance $0.001 \mathrm{~mm}$ from the front face of the metal layer. The incident electric vector is polarized parallel to the slit direction and must undergo a $90^{\circ}$ rotation in order to couple to the fields within the slit, thereby satisfying boundary conditions. In addition, the field along the length of the slit is also spatially quantized. The capacitive behavior is limited by the finite geometry of the slit. The response of the inductive and capacitive regions of the structure allows coupling of incident radiation to the surface and reradiation into the far field. The EM response of structure $\mathrm{C}$ on resonance is similar to that of sample B; however, fields are further en- hanced by a factor of 2 . Thus, it is confirmed that the transmission mechanism of mode 2 is the same for structures B and $\mathrm{C}$, with the difference in resonant frequency being attributed to the differing structure dimensions. The resonant frequency of each sample is highly dependent on the inductive and capacitive contributions of the structure; therefore, the thickness of the sample (capacitive part) and dimensions of the cut (inductive part) offer a high degree of tunability of the resonant frequency of the structure.

In summary, selective transmission of microwaves through a very thin array of subwavelength slits is presented. The examination of fields using a FEM model provides insight into the transmission mechanism, revealing one mode (that with $E$ fields perpendicular to the slits) to be due to diffractive coupling to a surface wave. A second mode transmits radiation with $E$ fields parallel to the slits and is due to the surface structure inducing surface currents, which create a zeroth-order Fabry-Pérot-type mode. This allows resonant transmission at wavelengths 100 times greater than the structure thickness. The frequency of transmission of this zerothorder Fabry-Pérot mode is readily controlled by the dimensions of the structure.

${ }^{1}$ J. A. Porto, F. J. Garcia-Vidal, and J. B. Pendry, Phys. Rev. Lett. 83, 2845 (1999).

${ }^{2}$ S. Astilean, Ph. Lalanne, and M. Palamaru, Opt. Commun. 175, 265 (2000).

${ }^{3}$ H. E. Went, A. P. Hibbins, J. R. Sambles, C. R. Lawrence, and A. P. Crick, Appl. Phys. Lett. 77, 2789 (2000).

${ }^{4}$ Y. Takakura, Phys. Rev. Lett. 86, 5601 (2001).

${ }^{5}$ A. P. Hibbins, J. R. Sambles, C. R. Lawrence, and D. M. Robinson, Appl. Phys. Lett. 79, 2844 (2001).

${ }^{6}$ A. P. Hibbins, J. R. Sambles, and C. R. Lawrence, Appl. Phys. Lett. 81, 4661 (2002).

${ }^{7}$ J. R. Suckling, A. P. Hibbins, M. J. Lockyear, T. W. Preist, J. R. Sambles, and C. R. Lawrence, Phys. Rev. Lett. 92, 147401 (2004).

${ }^{8}$ A. P. Hibbins, J. R. Sambles, C. R. Lawrence, and J. R. Brown, Phys. Rev. Lett. 92, 143904 (2004).

${ }^{9}$ J. R. Suckling, J. R. Sambles, and C. R. Lawrence, Phys. Rev. Lett. 95, 187407 (2005).

${ }^{10}$ HFSS, Ansoft Corporation, Pittsburgh, PA, USA. 